\title{
MiR-148a modulates HLA-G expression and influences tumor apoptosis in esophageal squamous cell carcinoma
}

\author{
QUAN CHEN $^{1}$, GUANGHUA LUO ${ }^{2}$ and XIAOYING ZHANG ${ }^{1}$ \\ ${ }^{1}$ Department of Cardiothoracic Surgery; ${ }^{2}$ Comprehensive Laboratory, The Third Affiliated \\ Hospital of Soochow University, Changzhou, Jiangsu 213003, P.R. China
}

Received October 20, 2016; Accepted June 6, 2017

DOI: 10.3892/etm.2017.5058

\begin{abstract}
Esophageal cancer (EC) is a common malignant tumor type, and esophageal squamous cell carcinoma (ESCC) accounts for the majority of EC cases. Previous studies have reported that microRNA ( $\mathrm{miR})-148 \mathrm{a}$ is downregulated in patients with recurrent EC. The human leukocyte antigen-G (HLA-G) is expressed to a high level in primary ESCC tissues and is associated with prognosis. A previous luciferase assay indicated that HLA-G is a target of miR-148a regulation. The aim of the current study was to investigate the expression level of miR-148a in primary ESCC. The regulatory role of miR-148a in HLA-G expression and cell proliferation in ESCC cells was also investigated. The relative expression level of miR-148a was compared between ESCC tumor tissues and adjacent normal tissues. The human ESCC cell line EC9706 was transfected with miR-148a mimic, non-homologous RNA duplex (negative control; NC) or empty vector (blank control; BC). Reverse transcription-quantitative polymerase chain reaction and western blot analysis were used to assess the level of HLA-G expression. The cells were stained with Annexin V-fluorescein isothiocyanate/propidium iodide and cell apoptosis was evaluated by flow cytometry. The level of miR-148a expression was significantly lower in primary ESCC tissues compared with adjacent normal tissues $(\mathrm{P}<0.01)$. In EC9706 cells transfected with miR-148a mimic, the rate of apoptosis was increased $\sim 13$-fold when compared with BC cells $(\mathrm{P}<0.01)$. Furthermore, the mRNA level of HLA-G was significantly reduced in cells transfected with miR-148a mimic $(\mathrm{P}<0.01)$. The protein levels of HLA-G were also notably decreased. Transfection with non-homologous RNA duplex did not influence the rate of cell apoptosis or expression of HLA-G when compared with the BC group. In conclusion, miR-148a was indicated to be involved in carcinogenesis in
\end{abstract}

Correspondence to: $\mathrm{Dr}$ Xiaoying Zhang, Department of Cardiothoracic Surgery, The Third Affiliated Hospital of Soochow University, 185 Juqian Road, Changzhou, Jiangsu 213003, P.R. China E-mail: xyzhang_cz@sina.com

Key words: esophageal squamous cell carcinoma, micoRNA-148a, human leukocyte antigen-G, apoptosis, tumor genesis primary ESCC through the regulation of HLA-G expression. The current results suggest that miR-148a is a potential biomarker of ESCC.

\section{Introduction}

Esophageal cancer (EC) is a common malignant tumor type originating from the esophageal mucosa (1). China has a high incidence of EC with a rate of 23.38/100,000 for males and $14.03 / 100,000$ for females (2). EC is the fifth most common type of cancer and esophageal squamous cell carcinoma (ESCC) accounts for $90 \%$ of all EC cases $(3,4)$. According to data from the National Central Cancer Registry of China, 477,900 new EC cases and 375,000 cases of mortality occurred in 2015 (5). The majority of EC patients are diagnosed at an advanced stage when the 5-year survival rate is $\sim 20 \%$, though early detected EC can be treated with endoscopic resection, and has a 5-year survival rate of up to $95 \%$ (6). Therefore, early detection and prompt intervention is an effective strategy to improve the prognosis of patients with EC and reduce the disease burden.

Compared with endoscopic biopsy, detection of serum tumor markers is a simple and minimally invasive method for early diagnosis (7). Circulating microRNAs (miRs) are stable in the blood and exhibit a high degree of tissue and tumor specificity, which makes them suitable as potential serum tumor markers (8). MiRs are also key regulators of gene transcription, and are frequently involved in the occurrence of cancers (9). In particular, miR-148a has been reported to be a tumor suppressor in multiple cancer types, including gastrointestinal (10), breast (11), ovarian (12), colorectal (13) and prostate cancer (14). Hummel et al (15) reported that miR-148a was downregulated in patients with recurrent EC. They also demonstrated that in EC cell lines, upregulation of miR-148a improved sensitivity to chemotherapy (16). However, whether miR-148a is downregulated in primary ESCC remains unclear.

Previous studies have demonstrated that the human leukocyte antigen-G (HLA-G) gene is one of the target genes regulated by miR-148a. Tan et al (17) identified that miR-148a could target HLA-G, and that differential expression of HLA-G interacted with the mother's asthma status to determine a child's risk of asthma. Manaster et al (18) confirmed by luciferase assay that miR-148a could downregulate HLA-G expression. A C/G polymorphism exists at position +3142 of 
the HLA-G gene, which is in the seed sequence of miR-148a; Manaster et al (18) identified that this polymorphism had no influence on HLA-G expression.

HLA-G is a member of the non-classical major histocompatibility complex class I antigens and serves a key function in maternal-fetal tolerance during pregnancy (19). Aberrant expression of HLA-G has been observed in multiple malignant cell types, which may be associated with the strategy of evading host immunosurveillance (20). Epithelial cells of the normal esophagus lack expression of HLA-G, while HLA-G is expressed to a high level in primary ESCC tissues and is associated with prognosis (21). Furthermore, the soluble isoforms of HLA-G in the plasma may be useful molecules in the differential diagnosis of patients with ESCC against healthy controls (22).

Based on previous reports, it was hypothesized that miR-148a may be differentially expressed in ESCC tissues, and this was investigated in the present study. The regulatory role of miR-148a on HLA-G expression and cell proliferation in ESCC cells was also investigated.

\section{Materials and methods}

Tissue samples. A total of 20 pairs of ESCC tumor tissues and normal adjacent tissues were used in the present study. Tissues were isolated from newly diagnosed ESCC patients who had not received chemotherapy or radiotherapy. A total of 20 patients ( 8 male, 12 female; mean age, 57.6 years) were recruited from the Department of Cardiothoracic Surgery in the Third Affiliated Hospital of Soochow University (Changzhou, China) from March 2012 to June 2013. The diagnosis was confirmed based on histological examination according to the American Joint Committee on Cancer 2010 TNM staging system for EC $(23,24)$. The samples were collected with written informed consent from the patients and stored in liquid nitrogen until assays were performed. The study protocol was approved by the Ethics Board of The Third Affiliated Hospital of Soochow University.

Cell line and culture. The human ESCC cell line EC9706 was purchased from American Type Culture Collection (Manassas, VA, USA). The cells were cultured in RPMI-1640 medium (Gibco; Thermo Fisher Scientific, Inc., Waltham, MA, USA) supplemented with $10 \%$ (V/V) fetal bovine serum (Gibco; Thermo Fisher Scientific, Inc.) in a humidified hood with 5\% $\mathrm{CO}_{2}$ at $37^{\circ} \mathrm{C}$.

Transfection of miR-148a mimic. The cells were seeded into 96-well plates to $60 \%$ confluency in RMPI-1640 media supplemented with 10\% FBS (antibiotic-free medium) for $24 \mathrm{~h}$ at $37^{\circ} \mathrm{C}$ prior to transfection. Lipofectamine 2000 reagent (Invitrogen; Thermo Fisher Scientific, Inc.) was used to transfect 1,000-10,000 cells/well with $50 \mathrm{nM}$ miR-148a mimic. The experiment was performed in triplicate for each group. Non-homologous RNA duplex was used as a negative control (NC) and empty vector was used as a blank control (BC). The nucleotides were chemically synthesized by Shanghai GenePharma Co., Ltd. (Shanghai, China). The nucleotide sequences used were as follows: For miR-148a mimic, forward, 5'-UCAGUGCAUGACAGAACUUGG-3' and reverse, 5'-AAG
TTCUGUCAUGCACUGAUU-3'; and for NC, forward, 5'-UUCUCCGAACGUGUCACGUTT-3' and reverse, 5'-ACG UGACACGUUCGGAGAATT-3'. The cells were harvested for reverse transcription-quantitative polymerase chain reaction (RT-qPCR), western blot analysis and apoptosis assays $72 \mathrm{~h}$ after transfection under $37^{\circ} \mathrm{C}$.

$R N A$ extraction and $R T-q P C R$. Total RNA was extracted from tissues and cells using TRIzol reagent (Invitrogen; Thermo Fisher Scientific, Inc.) according to the manufacturer's protocol. Synthesis of cDNA was performed using a PrimeScript II First Strand cDNA Synthesis kit (Takara Biotechnology Co., Ltd., Dalian, China). Target gene DNA was amplified with specific primers and a Fast SYBR Green Master mix (Applied Biosystems; Thermo Fisher Scientific, Inc.). Mature miR-148a, U6 control and HLA-G were detected using the primers reported previously (14). The primers for the internal control GAPDH were: Forward, 5'-AGCGCGTGCCTTATACCA AG-3' and reverse, 5'-GCCGCTCAGAGAGATTCGT-3'. All amplifications were conducted with a 7500 Real-Time PCR system (Applied Biosystems; Thermo Fisher Scientific, Inc.). The thermocycling conditions were as follows: $94^{\circ} \mathrm{C}$ for $1 \mathrm{~min}$, followed by 45 cycles of $94^{\circ} \mathrm{C}$ for $20 \mathrm{sec}, 58^{\circ} \mathrm{C}$ for $30 \mathrm{sec}$ and $72^{\circ} \mathrm{C}$ for $30 \mathrm{sec}$. Relative mRNA expression was calculated using the $\Delta \Delta \mathrm{C}_{\mathrm{q}}$ method as described previously (25).

Western blot analysis. Cells were lysed with RIPA lysis buffer (R0020, Beijing Solarbio Science and Technology Co., Ltd). Protein was isolated by centrifuging the sample at $20,000 \mathrm{x} \mathrm{g}$ at $4^{\circ} \mathrm{C}$ for $15 \mathrm{~min}$. Protein concentration was determined using a BCA Protein assay kit (Beyotime Institute of Biotechnology, Haimen, China). Each lane was filled with $1 \mathrm{ng}$ protein and $10 \mu \mathrm{l}$ 6x SDS loading dye. Proteins were separated on $12.5 \%$ SDS-PAGE gels and transferred to polyvinylidene difluoride membranes (EMD Millipore, Billerica, MA, USA. The membrane was blocked with 5\% skimmed milk at room temperature for $1 \mathrm{~h}$, then probed with monoclonal mouse anti-HLA-G (sc-21799; Santa Cruz Biotechnology, Inc., Dallas, TX, USA; 1:1,000), or monoclonal mouse anti-GAPDH (sc-32233; Santa Cruz Biotechnology, Inc.; $1: 1,000)$ for $1 \mathrm{~h}$ at room temperature. The secondary antibody used was horseradish peroxidase-conjugated rabbit anti-mouse Immunoglobulin G antibody (6120-05; SouthernBiotech, Birmingham, AL, USA; 1:2,000) at room temperature for $45 \mathrm{~min}$. Signals were detected using an enhanced chemiluminescence kit (BH4004, Shanghai Ke Min Biotechnology Co., Ltd., Shanghai, China).

Apoptosis assay. Cells $\left(\sim 5 \times 10^{5}\right)$ were harvested and washed with phosphate-buffered saline. Cells were stained with Annexin V-fluorescein isothiocyanate (SouthernBiotech) for $5 \mathrm{~min}$ at room temperature. A final concentration of $10 \mu \mathrm{g} / \mathrm{ml}$ propidium iodide was added for $30 \mathrm{~min}$ at $4^{\circ} \mathrm{C}$, away from light, then flow cytometry was performed. Flow cytometry results were analyzed using CytoSpec version 7 (Purdue University Cytometry Laboratories, West Lafayette, IN, USA).

Bioinformatics prediction. TargetScan version 7.1 (www.targetscan.org/vert_71) online software was used to predict the miRNA that could target HLA-G. 


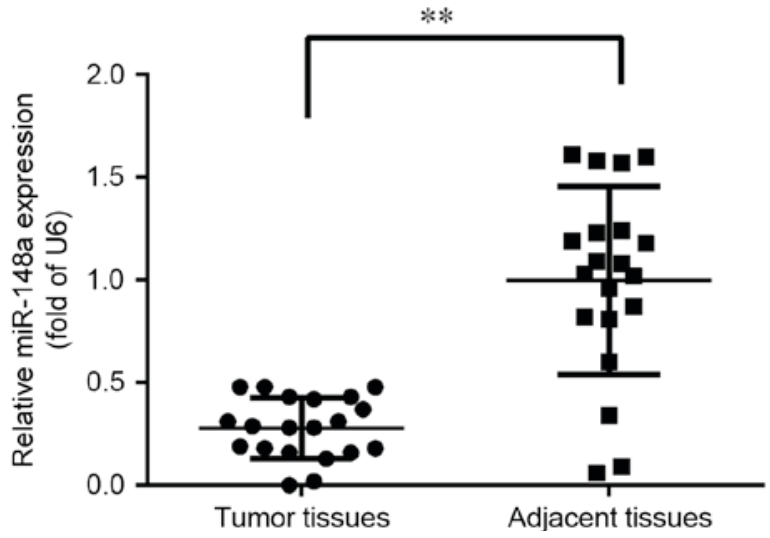

Figure 1. MiR-148a expression in primary ESCC tumor tissue. Expression of miR-148a was quantified in 20 paired ESCC and adjacent normal tissues. The expression level of miR-148a was normalized to that of U6. Error bars represent the standard deviation. ${ }^{* *} \mathrm{P}<0.01$ as indicated. ESCC, esophageal squamous cell carcinoma; miR, microRNA.

Statistical analysis. Results were analyzed using SPSS 16.0 software (SPSS, Inc., Chicago, IL, USA). Expression data regarding patients were expressed as the median (with maximum-minimum and interquartile range), and all other continuous variables were expressed as the mean \pm standard deviation. In vitro experiments were repeated at least three times. Comparisons among groups were analyzed using one-way analysis of variance and the least significant difference post hoc test. All tests were two-tailed and $\mathrm{P}<0.05$ was considered to indicate a statistically significant difference.

\section{Results}

Analysis of miR-148a expression in ESCC. To investigate the tumor-specific expression pattern of miR-148a, the expression of miR-148a was assessed in 20 pairs of primary ESCC tissues and matched adjacent normal tissues. As depicted in Fig. 1, expression of miR-148a was significantly reduced to $\sim 30 \%$ in the tumor tissue samples when compared with normal adjacent tissues $(\mathrm{P}<0.01)$. Therefore, a lower level of miR-148a expression was indicated to occur in ESCC.

Effect of miR-148a on HLA-G expression. Previous studies have indicated that HLA-G expression level may be regulated by miR-148a, and that miR-148b and HLA-G were upregulated in primary ESCC tissues $(13,16)$. Based on these reports, it was hypothesized that miR-148a may contribute to the proliferation of ESCC cells by regulating HLA-G expression. Therefore, the ESCC cell line EC9706 was transfected with miR-148a mimic to identify the potential regulatory role of miR-148a.

Position 376-382 of the HLA-G gene 3'-untranslated region is predicted as the binding site of miR-148a according to the TargetScan, as depicted in Fig. 2A. RT-qPCR confirmed that the level of HLA-G mRNA decreased following transfection with miR-148a mimic (Fig. 2B). Compared with cells transfected with nonsense (NC) or empty (BC) vector, the transcription level of HLA-G was significantly decreased (to a relative expression of $\sim 28 \%$ ) in cells transfected with miR-148a mimic $(\mathrm{P}<0.01$ vs. $\mathrm{BC})$. Accordingly, western blot analysis demonstrated that the level of HLA-G protein was reduced in
A hsa-miR-148a: 3'-UCAGCCUCACAGAGUCUUGAAA-5'
| I I | | | | |
HLA-G 3'UTR: 5'-UAAAUGGAAGACAUGAGACUUU-3'

B

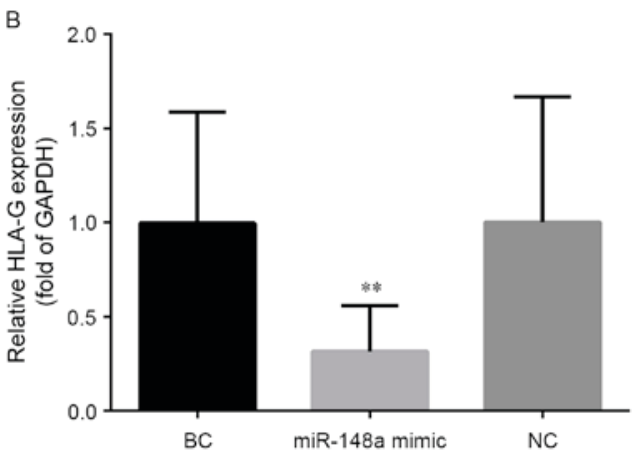

C

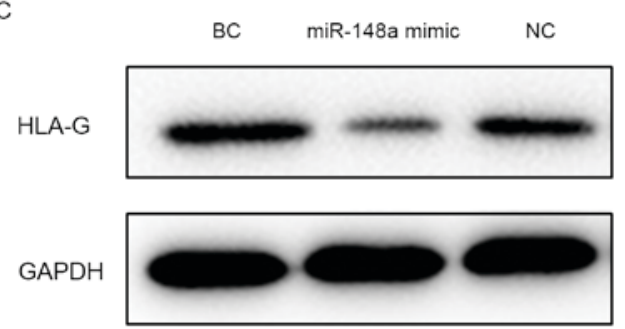

Figure 2. MiR-148a regulates HLA-G expression in the EC9706 cell line. (A) Position 376-382 of the HLA-G 3'UTR is a putative target site of miR-148a. (B) Relative mRNA level of HLA-G in cells transfected with miR-148a mimic or control vectors (NC or $\mathrm{BC}$ ). The expression level of HLA-G mRNA was normalized to that of GAPDH. (C) Protein level of HLA-G detected by western blotting in cells transfected with miR-148a mimic or control vectors. GAPDH was used as an internal control. ${ }^{* *} \mathrm{P}<0.01$ vs. BC group. UTR, untranslated region; miR, microRNA; NC, negative control; BC, blank control; HLA-G, human leukocyte antigen-G.
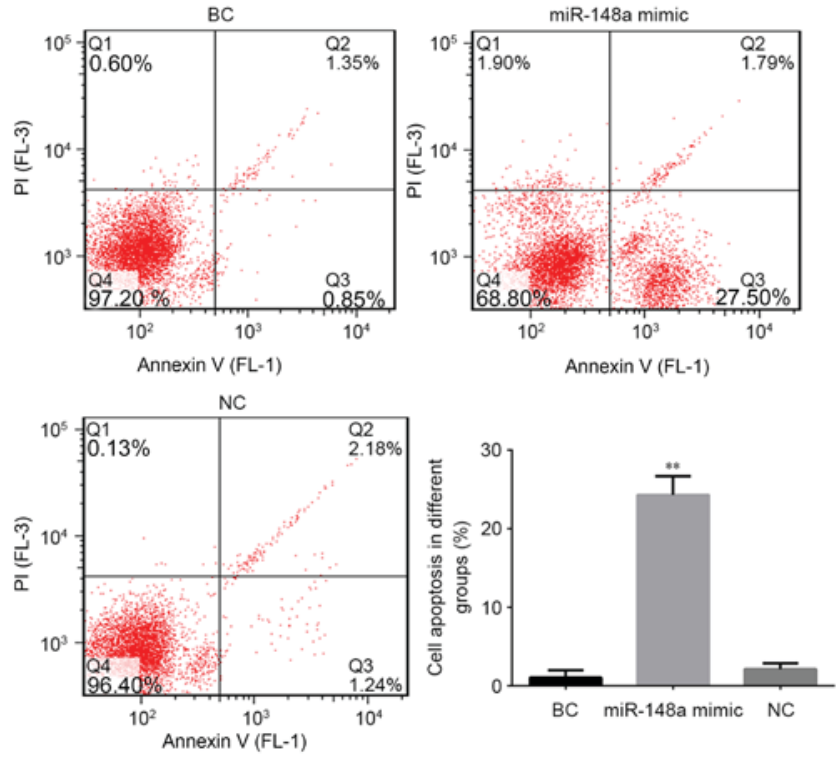

Figure 3. MiR-148a increases the rate of apoptosis in EC9706 cells. Cells were transfected with miR-148a mimic or control vectors (NC or BC). Cells $\left(\sim 5 \times 10^{5}\right.$ per treatment) were stained with Annexin V-fluorescein isothiocyanate and propidium iodide followed by flow cytometry analysis. ${ }^{* * *} \mathrm{P}<0.01 \mathrm{vs}$. BC group. miR, microRNA; NC, negative control; BC, blank control; Q1-4, quartiles 1-4. Percentages in quartiles 1-4 represent cells stained as annexin $\mathrm{V} / \mathrm{PI}^{+}$(necrotic cells), annexin $\mathrm{V}^{+} / \mathrm{PI}^{+}$(late apoptotic), annexin $\mathrm{V}^{-} / \mathrm{PI}^{-}$(alive cells) and annexin $\mathrm{V}^{+} / \mathrm{PI}^{-}$(early apoptotic), respectively. 
cells transfected with miR-148a mimic when compared with NC or BC cells (Fig. 2C). These results confirmed that HLA-G expression was regulated by miR-148a.

Effect of miR-148a on cell apoptosis. To further demonstrate whether miR-148a regulated the growth of cells, the rates of cell apoptosis were compared between the different cell groups. As depicted in Fig. 3, compared with the BC group, transfection with miR-148a significantly increased the rate of apoptosis in EC9706 cells $(\mathrm{P}<0.01)$; the apoptotic rate was $\sim 13$-fold higher in the cells transfected with miR-148a mimic. Rates of apoptosis did not differ between the NC and BC groups, indicating that the increase in apoptosis was due to transfection with miR-148a mimic. Collectively these results suggest that miR-148a may trigger apoptosis in ESCC cells. This pro-apoptotic function may explain the low expression of miR-148a and overexpression of its target HLA-G in ESCC tissues.

\section{Discussion}

Since the identification of miRs, large amounts of data have demonstrated the critical role of miRs in numerous biological processes including the occurrence, development and differentiation of cancers (26). Downregulation of miR-148a has been reported in multiple cancer types. In gastrointestinal cancer tissues, the level of miR-148a expression was inversely correlated with tumor size and cholecystokinin B receptor was identified as a potential target (10). It has also been reported that miR-148a may promote the proliferation of gastric cancer cells by directly targeting p27, as a key inhibitor of the cell cycle (27). Another study demonstrated that upregulation of miR-148a suppressed gastric cancer cell invasion in vitro and lung metastasis formation in vivo by downregulating the potential metastasis promoter Rho-associated coiled-coil containing protein kinase 1 (28). Furthermore, upregulation of miR-148a induced apoptosis in multiple colorectal cancer cell lines, and Bcl-2 was confirmed as its direct target (13). MiR-148a has further been implicated in hepatitis B-associated hepatocellular carcinoma through its targeting of the tumor suppressor, phosphatase and tensin homolog (29). Decreased expression of miR-148a has also been correlated with recurrent EC (15). Similarly, the present study observed that decreased expression of miR-148a was correlated with the occurrence of primary ESCC. To the best of our knowledge, this is the first report of a relationship between miR-148a and primary ESCC tissues.

Among all the reported target genes of miR-148a, HLA-G was chosen for further analysis in the current study, due to previous indications that HLA-G is expressed to a high level in primary ESCC tissues and may be regulated by miR-148a and miR-148b (18,21). Further in vitro experiments were performed that focused on miR-148a. By transfection with miR-148a mimic, it was confirmed that the expression of HLA-G was reduced by miR-148a in ESCC cells. These results indicated that upregulation of HLA-G may be due to a low level of miR-148a in ESCC.

HLA-G was first cloned and sequenced by Geraghty et al (30). HLA-G may be translated into seven isomers including transmembrane types (HLA-G1 to -G4) and soluble types (HLA-G5 to -G7) (31). It is generally accepted that HLA-G is an immune tolerance molecule that prevents maternal-fetal assaults through the inhibition of T- and natural killer cell-mediated cytolysis (32). Other inhibitory effects of HLA-G may include the induction of apoptosis of activated $\mathrm{CD}^{+} \mathrm{T}$-cells, downregulation of interferon secretion and induction of the Th2 cytokine profile (33). As a consequence of miR-148a downregulation, the increased expression of HLA-G by ESCC cells is likely to be an evasion strategy against the anti-tumor response.

It has been reported that an aberrant methylation status of HLA-G may be involved in the pathogenesis of cancers. In ovarian tumors, upregulation of HLA-G was associated with spontaneous demethylation in the HLA-G promoter region, and repression of the HLA-G gene could be reversed by demethylation (34). Similar epigenetic modification was observed in other tumor cell lines, including JEG-3 and Tera-2 $(35,36)$. DNA methyltransferase-1 (DNMT1) and DNMT3b are the key enzymes in mammalian cells that maintain DNA methylation, and both enzyme genes are direct targets of miR-148a $(37,38)$. Therefore, miR-148a may regulate HLA-G expression via a dual function: By direct silencing of the HLA-G gene and methylation regulation of the HLA-G promoter through DNMT1 and DNMT3b. However, this speculation requires further study.

Some limitations of this research should be mentioned. Although an association between miR-148a expression and primary ESCC tissues was reported, this result was observed in only 20 pairs of tumor tissues and adjacent tissues. The relationship between miR-148a expression and the clinicopathological characteristics and histologic grade of ESCC was not studied due to the small sample size. Ideally, a luciferase activity assay would have been performed to confirm the direct binding between miR-148a and HLA-G. However, since Manaster et al (18) provided this data previously, this assay was not performed in the present study.

In conclusion, the present study observed that miR-148a was involved in the carcinogenesis of primary ESCC and induced ESCC cell apoptosis by regulating HLA-G expression. The results suggest that miR-148a is a potential biomarker of ESCC.

\section{References}

1. Napier KJ, Scheerer M and Misra S: Esophageal cancer: A review of epidemiology, pathogenesis, staging workup and treatment modalities. World J Gastrointest Oncol 6: 112-120, 2014.

2. Chen WS, Zheng R, Zhang S, Zhao P, Zeng H, Zou X and He J: Annual report on status of cancer in China, 2010. Chin J Cancer Res 26: 48-58, 2014.

3. Arnold M, Soerjomataram I, Ferlay J and Forman D: Global incidence of oesophageal cancer by histological subtype in 2012. Gut 64: 381-387, 2015.

4. Chen W, Zheng R, Baade PD, Zhang S, Zeng H, Bray F, Jemal A, Yu XQ and He J: Cancer statistics in China, 2015. CA Cancer J Clin 66: 115-132, 2016.

5. Chen W, He Y, Zheng R, Zhang S, Zeng H, Zou X and He J: Esophageal cancer incidence and mortality in China, 2009. J Thorac Dis 5: 19-26, 2013.

6. Merkow RP, Bilimoria KY, Keswani RN, Chung J, Sherman KL, Knab LM, Posner MC and Bentrem DJ: Treatment trends, risk of lymph node metastasis, and outcomes for localized esophageal cancer. J Natl Cancer Inst 106: pii: dju133, 2014.

7. Andrew C, Fergus N and Timothy U: Strategies to improve outcomes in esophageal adenocarcinoma. Expert Rev Anticancer Ther 14: 677-687, 2014.

8. Cheng GF: Circulating miRNAs: Roles in cancer diagnosis, prognosis and therapy. Adv Drug Deliv Rev 81: 75-93, 2015. 
9. Calin GA, Sevignani C, Dumitru CD, Hyslop T, Noch E Yendamuri S, Shimizu M, Rattan S, Bullrich F, Negrini M and Croce CM: Human microRNA genes are frequently located at fragile sites and genomic regions involved in cancers. P Natl Acad Sci USA 101: 2999-3004, 2004.

10. Chen Y, Song Y, Wang Z, Yue Z, Xu H, Xing C and Liu Z: Altered expression of miR-148a and miR-152 in gastrointestinal cancers and its clinical significance. J Gastrointest Surg 14: 1170-1179, 2010.

11. Xu Q, Jiang Y, Yin Y, Li Q, He J, Jing Y, Qi YT, Xu Q, Li W, $\mathrm{Lu}$ B, et al: A regulatory circuit of miR-148a/152 and DNMT1 in modulating cell transformation and tumor angiogenesis through IGF-IR and IRS1. J Mol Cell Biol 5: 3-13, 2013.

12. Zhou X, Zhao F, Wang ZN, Song YX, Chang H, Chiang Y and Xu HM: Altered expression of miR-152 and miR-148a in ovarian cancer is related to cell proliferation. Oncol Rep 27: 447-454, 2012.

13. Zhang $\mathrm{H}, \mathrm{Li}$ Y, Huang Q, Ren $\mathrm{X}, \mathrm{Hu} \mathrm{H}$, Sheng $\mathrm{H}$ and Lai M: MiR-148a promotes apoptosis by targeting Bcl-2 in colorectal cancer. Cell Death Differ 18: 1702-1710, 2011.

14. Murata T, Takayama K, Katayama S, Urano T, Horie-Inoue K, Ikeda K, Takahashi S, Kawazu C, Hasegawa A, Ouchi Y, et al: miR-148a is an androgen-responsive microRNA that promotes LNCaP prostate cell growth by repressing its target CAND1 expression. Prostate Cancer Prostatic Dis 13: 356-361, 2010.

15. Hummel R, Hussey DJ, Michael MZ, Haier J, Bruewer M, Senninger N and Watson DI: MiRNAs and their association with locoregional staging and survival following surgery for esophageal carcinoma. Ann Surg Oncol 18: 253-260, 2011.

16. Hummel R, Watson DI, Smith C, Kist J, Michael MZ, Haier J and Hussey DJ: Mir-148a improves response to chemotherapy in sensitive and resistant oesophageal adenocarcinoma and squamous cell carcinoma cells. J Gastrointest Surg 15: 429-438, 2011.

17. Tan Z, Randall G, Fan J, Camoretti-Mercado B, Brockman-Schneider R, Pan L, Solway J, Gern JE, Lemanske RF, Nicolae D and Ober C: Allele-specific targeting of microRNAs to HLA-G and risk of asthma. Am J Hum Genet 81: 829-834, 2007.

18. Manaster I, Goldman-Wohl D, Greenfield C, Nachmani D, Tsukerman $\mathrm{P}$, Hamani $\mathrm{Y}$, Yagel $\mathrm{S}$ and Mandelboim $\mathrm{O}$ MiRNA-mediated control of HLA-G expression and function. PLoS One 7: e33395, 2012.

19. Carosella ED, Moreau P, LeMaoult $\mathrm{J}$ and Rouas-Freiss $\mathrm{N}$ : HLA-G: From biology to clinical benefits. Trends Immunol 29 $125-132,2008$.

20. Chang CC, Campoli M and Ferrone S: HLA class I antigen expression in malignant cells: Why does it not always correlate with CTL-mediated lysis? Curr Opin Immunol 16: 644-650, 2004

21. Yie SM, Yang H, Ye SR, Li K, Dong DD and Lin XM: Expression of HLA-G is associated with prognosis in esophageal squamous cell carcinoma. Am J Clin Pathol 128: 1002-1009, 2007.

22. Cao M, Yie SM, Liu J, Ye S, Xia D and Gao E: Plasma soluble HLA-G is a potential biomarker for diagnosis of colorectal, gastric, esophageal and lung cancer. Tissue Antigens 78: 120-128, 2011.
23. Rice TW: Esophageal cancer staging. Korean J Thorac Cardiovasc Surg 48: 157-163; 2015

24. Napier KJ, Scheerer M and Misra S: Esophageal cancer: A Review of epidemiology, pathogenesis, staging workup and treatment modalities. World J Gastrointest Oncol 6: 112-120, 2014.

25. Livak KJ and Schmittgen TD: Analysis of relative gene expression data using real-time quantitative PCR and the 2(-Delta Delta C(T)) method. Methods 25: 402-408, 2001

26. Berindan-Neagoe I, Monroig P, Pasculli B and Calin GA MicroRNAome genome: A treasure for cancer diagnosis and therapy. CA Cancer J Clin 64: 311-336, 2014.

27. Guo SL, Peng Z, Yang X, Fan KJ, Ye H, Li ZH, Wang Y, Xu XL, Li J, Wang YL, et al: miR-148a promoted cell proliferation by targeting p27 in gastric cancer cells. Int J Biol Sci 7: 567-574, 2011.

28. Zheng B, Liang L, Wang C, Huang S, Cao X, Zha R, Liu L, Jia D, Tian Q, Wu J, et al: Microrna-148a suppresses tumor cell invasion and metastasis by downregulating rock1 in gastric cancer. Clin Cancer Res 17: 7574-7583, 2011.

29. Yuan K, Lian Z, Sun B, Clayton MM, Ng Io and Feitelson MA Role of miR-148a in hepatitis B associated hepatocellular carcinoma. PLoS One 7: e35331, 2012.

30. Geraghty DE, Koller BH and Orr HT: A human major histocompatibility complex class I gene that encodes a protein with a shortened cytoplasmic segment. Proc Natl Acad Sci USA 84: 9145-9149, 1987.

31. Paul P, Adrian Cabestre FA, Ibrahim EC, Lefebvre S, Khalil-Daher I, Vazeux G, Quiles RM, Bermond F, Dausset J and Carosella ED: Identification of HLA-G7 as a new splice variant of the HLA-G mRNA and expression of soluble HLA-G5, -G6 and -G7 transcripts in human transfected cells. Hum Immunol 61: 1138-1149, 2000.

32. Rizzo R, Vercammen M, van de Velde H, Horn PA and Rebmann V: The importance of HLA-G expression in embryos, trophoblast cells and embryonic stem cells. Cell Mol Life Sci 68: 341-352, 2011.

33. Rouas-Freiss N, Moreau P, Ferrone S and Carosella D: HLA-G proteins in cancer: Do they provide tumor cells with an escape mechanism? Cancer Res 65: 10139-10144, 2005.

34. Menendez L, Walker LD, Matyunina LV, Totten KA, Benigno BB and McDonald JF: Epigenetic changes within the promoter region of the HLA-G gene in ovarian tumors. Mol Cancer 7: 1-11, 2008.

35. Moreau P, Mouillot G, Rousseau P, Marcou C, Dausset J and Carosella ED: HLA-G gene repression is reversed by demethylation. Proc Natl Acad Sci USA 100: 1191-1196, 2003.

36. Mouillot G, Marcou C, Rousseau P, Rouas-Freiss N, Carosella ED and Moreau P: HLA-G gene activation in tumor cells involves cis-acting epigenetic changes. Int J Cancer 113: 928-936, 2005

37. Braconi C, Huang N and Patel T: MicroRNA-dependent regulation of DNA methyltransferase-1 and tumor suppressor gene expression by interleukin- 6 in human malignant cholangiocytes. Hepatology 51: 881-890, 2010.

38. Duursma AM, Kedde M, Schrier M, Ie Dage C and Agami R miR-148 targets human DNMT3b protein coding region. RNA 14: 872-877, 2008. 